\section{Body Recognition in a Patient with Bilateral Primary Visual Cortex Lesions}

\section{To the Editor:}

When the primary visual cortex of a cerebral hemisphere is lesioned, the patient loses sight in the contralateral visual field. However, it has been shown that some patients are still capable of "recognizing" certain visual attributes of stimuli that are presented in their blind visual field and for which they have no conscious awareness of their presence. For example, a patient with a lesion in the right primary visual cortex and ensuing clinical blindness of the left visual field was able to discriminate above chance level the orientation of lines that were presented in his blind visual field and without acknowledged awareness of the stimuli (1). Recent neuroimaging findings in such patients reported extensive activations of extrastriate visual areas in the intact hemisphere in response to stimuli presented in the blind field $(2,3)$, as well as the formation of new fiber connections linking intact subcortical areas in the damaged hemisphere with extrastriate areas in the intact hemisphere (4). This suggests that spared object categorization for unseen stimuli is, at least in part, mediated by striate and extrastriate visual areas along the ventral stream of the intact hemisphere.

We investigated object recognition in a patient with cortical blindness over the entire visual field.

\section{Case Report}

\section{History}

A 58-year-old male physician suffered 2 occipital strokes in less than 2 months, leading to bilateral destruction of primary visual cortices (V1), more than 7 years before the current investigations. Clinically, he appears to be totally blind, and extensive behavioral and neuroimaging experiments could not provide evidence of perceptual awareness or functioning of primary visual cortex (5). Remarkably, he was able to avoid obstacles positioned in a corridor he walked through, showing that V1-independent neural pathways can still sustain sophisticated navigational functions and visuomotor integration (6). Possibly, alternative (subcortical) pathways exist to sustain egocentric visuospatial guidance of object-directed movements by activating the dorsal stream (the "where" stream). However, the residual functions following primary visual cortex destruction of the other main visual function (i.e., object recognition) sustained by the ventral stream (the "what" stream) (7) remain an open question.

\section{Behavioral Testing}

Visual Perimetry. A white circle $\left(1^{\circ}\right.$; luminance $\left.95 \mathrm{~cd} / \mathrm{m}^{2}\right)$ was presented for 300 milliseconds at each of 64 locations (16 per quadrant) against a dark background $\left(2 \mathrm{~cd} / \mathrm{m}^{2}\right)$. A similar procedure was performed with flickering stimuli $(20 \mathrm{~Hz})$. The patient did not detect any stimulus change, confirming his clinical blindness.
Object Recognition. Stimuli consisted of 120 images equally divided over five categories: faces, whole bodies (without faces), butterflies, cars and scrambled images. All categories were matched for size and luminance. The experiment was divided in five blocks. In each block, all 120 stimuli were presented singly at the center of the screen for 1500 milliseconds, their onset and offset announced by two different sounds. Instructions stated to guess whether the stimulus matched the target category of the respective block (e.g., face or no face). Per block, we calculated the number of correctly categorized target stimuli. He categorized human bodies significantly above chance level, whereas categorization of the other stimuli was at chance (correct categorization: human bodies, 13 of $24 ; p<.001$ by binomial test; faces, 6 of 24; butterflies, 7 of 24; cars 8 of 24; scrambles, 5 of 24 ; $p>$.09).

Imaging. To investigate the neural correlates of his preserved ability to categorize human bodies, we used functional magnetic resonance imaging and presented images of the same categories while he lay in the scanner and was instructed to keep his eyes open and fixate straight ahead. The stimulation protocol consisted of alternating fixation (24,000 milliseconds) and stimulation (20,000 milliseconds) blocks. During stimulation blocks, 10 stimuli of the same category were presented one by one for 1500 milliseconds with an interstimulus interval of 500 milliseconds. Five categories were presented: bodies, faces, butterflies, cars, and scrambled images. A passive exposure paradigm ensured that results were unaffected by top-down factors such as action execution or button press. The run was pseudo-randomized with four blocks of every stimulus category. Data were collected on a 3T TrioMRI scanner (Siemens Medical Solutions, Erlangen, Germany). An anatomical scan was acquired using a three-dimensional magnetization prepared rapid acquisition gradient echo T1-weighted sequence (repetition time/echo time/inversion time $=2.5 \mathrm{sec} / 2.9 \mathrm{msec} / 1.1 \mathrm{sec}$, field of view $=230 \mathrm{~mm}$, matrix $256 \times 256$, slice thickness = $.9 \mathrm{~mm})$. A T2*-weighted gradient echo type echo planar imaging sequence was applied for whole brain blood oxygen level-dependent sensitive magnetic resonance imaging (repetition time/echo time/flip $=2 \mathrm{sec} / 30 \mathrm{msec} / 85^{\circ}$, field of view $220 \mathrm{~mm}$, matrix $86 \times 86$, in plane resolution $2.5 \times 2.5 \mathrm{~mm}$, 32 contiguous $3-\mathrm{mm}$ axial slices with .45-mm gap). Preprocessing of the functional data included slice scan-time correction (cubic spline interpolation), three-dimensional motion correction (trilinear/sinc interpolation), and temporal filtering (high pass General Linear Model-Fourier of 2 sines/cosines). Functional data were transferred into Talairach space. Analysis was based on the General Linear Model, with each condition defined as a predictor. Threshold was set at $p<.05$, Bonferroni corrected for multiple comparisons.

Imaging Results. An area in the right occipitotemporal cortex bordering the lesion was more active when he was shown pictures of human bodies, compared with the other categories (Figure 1). This location corresponds to the extrastriate body area, which has been shown to process perception of the human body shape in normal subjects (8). Its critical involvement in body recognition is further substantiated by 

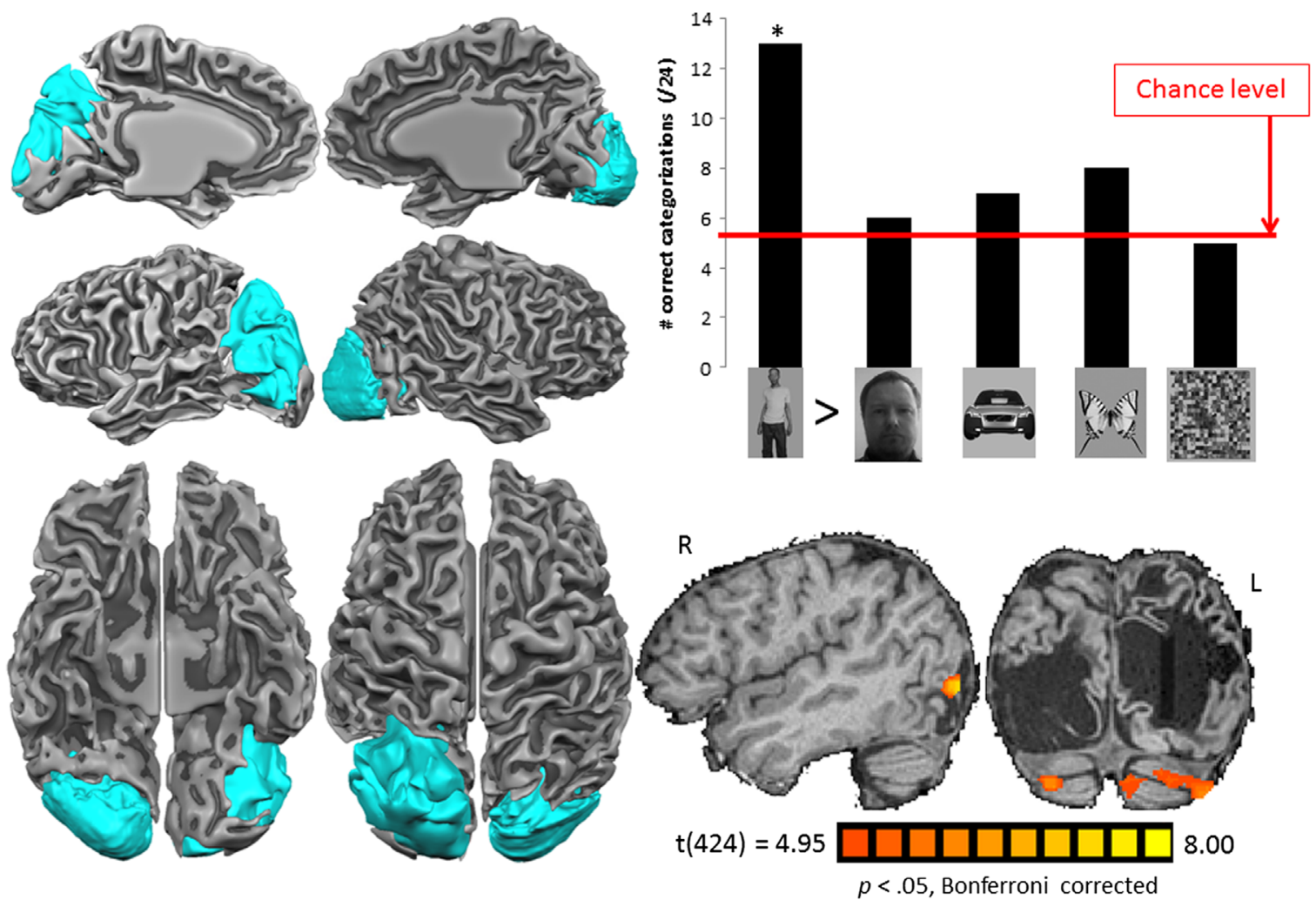

Figure 1. Imaging and behavioral results. The left panel shows a cortical reconstruction of the patient's brain with the lesions colored in turquois. Gyri are shown in light gray, sulci in dark gray. The top right panel shows the behavioral results indicating above-chance categorization of human body shape stimuli $\left({ }^{*} p<.001\right)$. The red line indicates chance level (4.8). The bottom right panel shows the statistical activation maps when comparing presentation of body stimuli with the other categories (threshold $p<.05$, Bonferroni corrected for multiple comparisons). Statistical color coding is shown on the bottom right.

studies showing impaired body shape recognition when extrastriate body area is virtually lesioned by means of transcranial magnetic stimulation (9). The neural pathways sustaining input to the ventral stream in the absence of V1 presumably rely largely on subcortical structures. In line with this, we observed that the bilateral posterior lobes of the cerebellum also responded more to bodies than to the other categories. This region has been associated with categorical perception (10), including body perception (11).

These results suggest a neural mechanism underlying visual object perception not accompanied by visual awareness. The results are in line with a recent report of object recognition without awareness in normal subjects (12) and additionally have therapeutic implications. Specific training programs for stimulating residual visual capacities after V1 damage were shown to be effective for complex motion perception (13) and grating discrimination (14). These results may provide an incentive for the development of cognitive rehabilitation programs (15) targeting some areas of object recognition.

Jan Van den Stock Marco Tamietto Alexis Hervais-Adelman Alan J. Pegna Beatrice de Gelder

\section{Acknowledgments and Disclosures}

JVdS is a postdoctoral researcher supported by Fonds Wetenschappelijk Onderzoek-Vlaanderen (Grant No. 1.5.072.13N). MT is supported by a Vid grant from The Netherlands Organization for Scientific Research (Grant No. 452-11-015) and by a FIRB - Futuro in Ricerca 2012 grant from the Italian Ministry of Education University and Research (Grant No. RBFR12F0BD). BdG is supported in part by National Initiative Brain \& Cognition (Grant No. 056-22-011) and FP7-FET-Open grants and by an Advanced European Research Counsil grant. AP is supported by the Swiss National Science Foundation (Grant No. 320030-144187). AH-A is supported by the Swiss National Science Foundation (Grant No. 320030-122085 awarded to Professor Narly Golestani). Functional mangnetic resonance imaging was performed at the Center for Biomedical Imaging of Geneva and Lausanne.

We thank artist and photographer G. Friedler for use of his photographs of bodies for this study.

The authors reported no biomedical financial interests or potential conflicts of interest.

\section{Article Information}

From the Brain and Emotion Laboratory Leuven (BELL) (JVdS, BDG), Division of Psychiatry, Department of Neurosciences, KU Leuven, Leuven, Belgium; Cognitive Neuroscience (MT, AJP, BdG), Faculty of Psychology and Neuroscience, Maastricht University, the Netherlands; Functional Brain Mapping Laboratory (AH-A), CMU; Laboratory of Experimental Neuropsychology (AH-A AJP), Neurology Clinic, Geneva University Hospitals; and Faculty of Psychology and Educational Science (AJP), University of Geneva, Geneva, Switzerland.

Authors JVdS and MT contributed equally to this work.

Address Correspondence to Beatrice de Gelder; E-mail: b.degelder@ maastrichtuniversity.nl. 


\section{References}

1. Weiskrantz L (2009): Blindsight: A Case Study Spanning 35 Years and New Developments. Oxford: Oxford University Press.

2. Van den Stock J, Tamietto M, Sorger B, Pichon S, Grezes J, de Gelder B (2011): Cortico-subcortical visual, somatosensory, and motor activations for perceiving dynamic whole-body emotional expressions with and without striate cortex (V1). Proc Natl Acad Sci U S A 108: 16188-16193.

3. Goebel R, Muckli L, Zanella FE, Singer W, Stoerig P (2001): Sustained extrastriate cortical activation without visual awareness revealed by fMRI studies of hemianopic patients. Vision Res 41:1459-1474.

4. Bridge H, Thomas O, Jbabdi S, Cowey A (2008): Changes in connectivity after visual cortical brain damage underlie altered visual function. Brain 131:1433-1444.

5. Pegna AJ, Khateb A, Lazeyras F, Seghier ML (2005): Discriminating emotional faces without primary visual cortices involves the right amygdala. Nat Neurosci 8:24-25.

6. de Gelder B, Tamietto M, van Boxtel G, Goebel R, Sahraie A, van den Stock J, et al. (2008): Intact navigation skills after bilateral loss of striate cortex. Curr Biol 18:R1128-R1129.

7. Goodale MA, Milner MA (2004): Sight Unseen: An Exploration of Conscious and Unconscious Vision. Oxford: Oxford University Press.
8. Downing PE, Jiang Y, Shuman M, Kanwisher N (2001): A cortical area selective for visual processing of the human body. Science 293: 2470-2473.

9. Urgesi C, Candidi M, lonta S, Aglioti SM (2007): Representation of body identity and body actions in extrastriate body area and ventral premotor cortex. Nat Neurosci 10:30-31.

10. Van den Stock J, Vandenbulcke M, Zhu Q, Hadjikhani N, de Gelder B (2012): Developmental prosopagnosia in a patient with hypoplasia of the vermis cerebelli. Neurology 78:1700-1702.

11. Sokolov AA, Gharabaghi A, Tatagiba MS, Pavlova M (2010): Cerebellar engagement in an action observation network. Cereb Cortex 20: 486-491.

12. Norman LJ, Heywood CA, Kentridge RW (2013): Object-based attention without awareness. Psychol Sci 24:836-843.

13. Huxlin KR, Martin T, Kelly K, Riley M, Friedman DI, Burgin WS, et al. (2009): Perceptual relearning of complex visual motion after V1 damage in humans. J Neurosci 29:3981-3991.

14. Sahraie A, Trevethan CT, MacLeod MJ, Murray AD, Olson JA, Weiskrantz L (2006): Increased sensitivity after repeated stimulation of residual spatial channels in blindsight. Proc Natl Acad Sci U S A 103:14971-14976.

15. Stoerig P (2008): Functional rehabilitation of partial cortical blindness? Restor Neurol Neurosci 26:291-303. 